University of Nebraska - Lincoln

DigitalCommons@University of Nebraska - Lincoln

Faculty Publications from the Department of Electrical \& Computer Engineering, Department Electrical and Computer Engineering

2011

\title{
On the Performance Limits of Cognitive MIMO Channels
}

\author{
Sami Akin \\ University of Nebraska-Lincoln, samiakin@huskers.unl.edu \\ M. Cenk Gursoy \\ University of Nebraska-Lincoln, gursoy@engr.unl.edu
}

Follow this and additional works at: https://digitalcommons.unl.edu/electricalengineeringfacpub

Part of the Electrical and Computer Engineering Commons

Akin, Sami and Cenk Gursoy, M., "On the Performance Limits of Cognitive MIMO Channels" (2011). Faculty Publications from the Department of Electrical and Computer Engineering. 160.

https://digitalcommons.unl.edu/electricalengineeringfacpub/160

This Article is brought to you for free and open access by the Electrical \& Computer Engineering, Department of at DigitalCommons@University of Nebraska - Lincoln. It has been accepted for inclusion in Faculty Publications from the Department of Electrical and Computer Engineering by an authorized administrator of DigitalCommons@University of Nebraska - Lincoln. 


\title{
On the Performance Limits of Cognitive MIMO Channels
}

\author{
Sami Akin and Mustafa Cenk Gursoy \\ Department of Electrical Engineering \\ University of Nebraska-Lincoln \\ Lincoln, NE 68588 \\ Email: samiakin@huskers.unl.edu, gursoy@engr.unl.edu
}

\begin{abstract}
In this paper, throughput of cognitive multiple-input multiple-output (MIMO) systems operating under quality-ofservice $(\mathrm{QoS})$ constraints is studied. It is assumed that transmission power and the covariance of the input signal vector are varied depending on the sensed activities of primary users in the system. Considering the reliability of the transmission and channel sensing results, a state-transition model is provided. Effective capacity is determined, and expressions for the first and second derivatives of the effective capacity are obtained at $S N R=0$. The minimum bit energy requirements in the presence of QoS limitations are identified. Numerical results are provided.
\end{abstract}

\section{INTRODUCTION}

MIMO communication schemes have attracted much interest from researchers in both academia and industry as they enhance the communication performance and quality thorough improvements in transmission rates and/or diversity without any increase in bandwidth and transmit power. Therefore, there have been numerous studies conducted concerning MIMO channels. In most studies, Shannon capacity formulation is considered as the performance metric (see e.g., [1]-[3] and references therein). Recently, effective capacity of MIMO channels have been studied in order to identify the performance limits in the presence of QoS constraints. More specifically, effective capacity defined in [4] is used as a throughput metric when the transmitters operate under buffer constraints. In [5], the maximization of the effective capacity is considered in a single-user multi-antenna system with covariance knowledge. In [6], effective capacity of a class of multiple-antenna wireless systems subject to Rayleigh flat fading is studied. In [7], effective capacity of MIMO systems is analyzed in a general setting under various assumptions on the channel knowledge, and performance in low- and high signalto-noise ratio (SNR) regimes is investigated.

In addition to MIMO techniques, cognitive radio has emerged in recent years as an advanced communication scheme that enables wireless systems to utilize spectrum white spaces and use the available spectrum more efficiently. A more detailed description of cognitive radio systems and models can be found in [8] and [9]. Furthermore, an analysis of multiple-antenna cognitive channels can be found in [10]. Cognitive users generally first sense the channel and subsequently adapt their transmission strategies depending on the policies and power interference limits of primary users. Therefore, sensing the activities of primary users is a vital issue that has been studied and analyzed since the cognitive radio concept was proposed [11], [12]. In our previous studies, we considered a cognitive radio model in which secondary user transmits with two different transmission rates and power levels depending on the activities of primary users under QoS constraints. In [13], the secondary transmitter senses only one channel and then depending on the channel sensing results, it chooses its transmission policy. In [14], effective capacity limits of a cognitive radio model is analyzed with imperfect channel side information (CSI) at the transmitter and the receiver.

In this paper, we focus on cognitive MIMO systems operating under QoS constraints. We investigate the cognitive MIMO performance in the presence of interference from the primary users, and consider two different transmission policies depending on the activities of primary users. We study the low-power regime and identify the impact of QoS limitations on the energy efficiency. This analysis is conducted for a general cognitive MIMO link model in which fading coefficients have arbitrary distributions and are correlated.

\section{Channel Model And Power Constraint}

We consider a cognitive MIMO channel model and assume that the secondary transmitter and receiver are equipped with $M$ and $N$ antennas, respectively. In a flat fading channel, we can express the channel input-output relation as

$$
\mathbf{y}=\mathbf{H x}+\mathbf{n}+\mathbf{s}
$$

if the primary users are active in the channel, and as

$$
\mathbf{y}=\mathbf{H x}+\mathbf{n}
$$

if the primary users are absent. Above, $\mathbf{x}$ denotes the $M \times 1$ dimensional transmitted signal vector, and $\mathbf{y}$ denotes the $N \times 1$ dimensional received signal vector. In (1) and (2), $\mathbf{n}$ is an $N \times 1$ dimensional zero-mean Gaussian random vector with covariance matrix $\mathbb{E}\left\{\mathbf{n} \mathbf{n}^{\dagger}\right\}=\sigma_{n}^{2} \mathbf{I}$ where $\mathbf{I}$ is the identity matrix. Hence, the components of $\mathbf{n}$ are independent. In (1), $\mathbf{s}$ is an $N \times 1$ dimensional vector of the sum of active primary users' faded signals arriving at the secondary receiver. Considering that the vector $\mathbf{s}$ can have correlated components, we express its covariance matrix as $\mathbb{E}\left\{\mathbf{s s}^{\dagger}\right\}=N \sigma_{s}^{2} \mathbf{K}_{s}$ where $\sigma_{s}^{2}$ is the variance of each component of $\mathbf{s}$ and $\operatorname{tr}\left(\mathbf{K}_{s}\right)=1$. Finally, in (1) and (2), $\mathbf{H}$ denotes the $N \times M$ dimensional random channel 
matrix whose components are the fading coefficients between the corresponding antennas at the secondary transmitting and receiving ends. We consider a block-fading scenario and assume that the realization of the matrix $\mathbf{H}$ remains fixed over a block duration of $T$ seconds and changes independently from one block to another.

We assume that the secondary users initially perform channel sensing to detect the activities of primary users, and then depending on the channel sensing results, they choose the transmission strategy. More specifically, if the channel is sensed as busy, the transmitted signal vectors is $\mathbf{x}_{1}$. Otherwise, the signal is $\mathbf{x}_{2}$. In order to control the interference caused to the primary users, the average transmission power levels can be different. The average energy constraint on the channel input is

$$
\mathbb{E}\left\{\left\|\mathbf{x}_{1}\right\|^{2}\right\} \leq \frac{P_{1}}{B}
$$

if the channel is sensed as busy. On the other hand, if the channel is detected to be idle, the energy constraint becomes

$$
\mathbb{E}\left\{\left\|\mathbf{x}_{2}\right\|^{2}\right\} \leq \frac{P_{2}}{B}
$$

In (3) and (4), $B$ is the bandwidth of the system.

Directionality of the transmitted signal vectors might also be different depending on the channel sensing results. We define the normalized input covariance matrices of $\mathbf{x}_{1}$ and $\mathbf{x}_{2}$ as

$$
\mathbf{K}_{x_{1}}=\frac{\mathbb{E}\left\{\mathbf{x}_{1} \mathbf{x}_{1}^{\dagger}\right\}}{P_{1} / B} \quad \text { and } \quad \mathbf{K}_{x_{2}}=\frac{\mathbb{E}\left\{\mathbf{x}_{2} \mathbf{x}_{2}^{\dagger}\right\}}{P_{2} / B} .
$$

Note that the trace of normalized covariance matrices are bounded by

$$
\operatorname{tr}\left(\mathbf{K}_{x_{1}}\right) \leq 1 \quad \text { and } \operatorname{tr}\left(\mathbf{K}_{x_{2}}\right) \leq 1
$$

We consider a practical scenario in which errors such as missdetections and false-alarms possibly occur in channel sensing. We denote the correct-detection and false-alarm probabilities by $P_{d}$ and $P_{f}$, respectively. We note the following two cases. When the primary users are active and this activity is sensed correctly (which happens with probability $P_{d}$ ), then the secondary users transmit with average power $P_{1}$. On the other hand, if the primary user activity is missed in sensing (which occurs with probability $1-P_{d}$ ), the secondary users send the information with average power $P_{2}$. In both cases, primary users experience interference. In order to limit the average interference, we impose the following power constraint

$$
P_{d} P_{1}+\left(1-P_{d}\right) P_{2} \leq P,
$$

Now, we define the average signal-to-noise ratio (SNR) as

$$
\mathrm{SNR}=\frac{P / B}{\mathbb{E}\left\{\|\mathbf{n}\|^{2}\right\}}=\frac{P}{N B \sigma_{n}^{2}}
$$

Also, we assume $P_{1}=\mu P$ and $P_{2}=\nu P$ where $\mu$ and $\nu$ are some positive numbers, and rewrite $(7)$ as

$$
P_{d} \mu P+\left(1-P_{d}\right) \nu P \leq P
$$

obtaining

$$
P_{d} \mu+\left(1-P_{d}\right) \nu \leq 1
$$

Note that, to maximize the channel throughput, we have to choose optimal $\mu$ and $\nu$ values.

\section{State Transition Model And Channel THROUGHPUT METRICS}

\section{A. State Transition Model}

We assume that both the secondary transmitter and receiver have perfect CSI and hence perfectly know the instantaneous values of $\{\mathbf{H}\}$ in each transmission block. More specifically, the secondary transmitter sends the information with optimized data vectors. Moreover, depending on channel sensing results and their correctness, we have four scenarios:

1) Channel is busy, detected as busy (correct detection), with probability $\rho P_{d}$,

2) Channel is busy, detected as idle (miss-detection), with probability $\rho\left(1-P_{d}\right)$,

3) Channel is idle, detected as busy (false alarm), with probability $(1-\rho) P_{f}$,

4) Channel is idle, detected as idle (correct detection), with probability $(1-\rho)\left(1-P_{f}\right)$.

Above, $\rho$ is the probability of channel being actually busy, and $P_{d}$ is the probability of detection, and $P_{f}$ is the probability of false alarm.

Using the notation $\mathbb{E}\left\{(\mathbf{s}+\mathbf{n})(\mathbf{s}+\mathbf{n})^{\dagger}\right\}=\mathbb{E}\left\{\mathbf{s s}^{\dagger}\right\}+\mathbb{E}\left\{\mathbf{n} \mathbf{n}^{\dagger}\right\}=$ $\sigma_{n}^{2} \mathbf{K}_{z}$ where $\operatorname{tr}\left(\mathbf{K}_{z}\right)=\frac{N\left(\sigma_{s}^{2}+\sigma_{n}^{2}\right)}{\sigma_{n}^{2}}$, we can express the instantaneous channel capacities in the above four scenarios as follows:

$$
\begin{aligned}
& C_{1}=B \max _{\substack{\mathbf{K}_{x_{1}} \succeq 0 \\
\operatorname{tr}\left(\mathbf{K}_{x_{1}}\right) \leq 1}} \log _{2} \operatorname{det}\left[\mathbf{I}+\mu N \operatorname{SNR} \mathbf{H} \mathbf{K}_{x_{1}} \mathbf{H}^{\dagger} \mathbf{K}_{z}^{-1}\right], \\
& C_{2}=B \max _{\substack{\mathbf{K}_{x_{2}} \succeq 0 \\
\operatorname{tr}\left(\mathbf{K}_{x_{2}}\right) \leq 1}} \log _{2} \operatorname{det}\left[\mathbf{I}+\nu N \operatorname{SNR} \mathbf{H K} \mathbf{K}_{x_{2}} \mathbf{H}^{\dagger} \mathbf{K}_{z}^{-1}\right], \\
& C_{3}=B \max _{\substack{\mathbf{K}_{x_{1}} \succeq 0 \\
\operatorname{tr}\left(\mathbf{K}_{x_{1}}\right) \leq 1}} \log _{2} \operatorname{det}\left[\mathbf{I}+\mu N \operatorname{SNR} \mathbf{H} \mathbf{K}_{x_{1}} \mathbf{H}^{\dagger}\right] \\
& C_{4}=B \max _{\substack{\mathbf{K}_{x_{2}} \succeq 0 \\
\operatorname{tr}\left(\mathbf{K}_{x_{2}}\right) \leq 1}} \log _{2} \operatorname{det}\left[\mathbf{I}+\nu N \operatorname{SNR} \mathbf{H K} \mathbf{K}_{x_{2}} \mathbf{H}^{\dagger}\right]
\end{aligned}
$$

We note that since $\mathbf{K}_{z}$ is a positive definite matrix and its eigenvalues are greater than or equal to $1, \mathbf{K}_{z_{2}}^{-1}$ is a positive definite matrix with eigenvalues $1 \geq \lambda_{i} \geq \frac{\sigma_{n}^{2}}{N\left(\sigma_{n}^{2}+\sigma_{s}^{2}\right)}$.

The secondary transmitter is assumed to send the data at two different rates depending on the sensing results. If the channel is detected as busy, the transmission rate is

$$
r_{1}=B \max _{\substack{\mathbf{K}_{x_{1}} \succeq 0 \\ \operatorname{tr}\left(\mathbf{K}_{x_{1}}\right) \leq 1}} \log _{2} \operatorname{det}\left[\mathbf{I}+\mu N \operatorname{SNR} \mathbf{H} \mathbf{K}_{x_{1}} \mathbf{H}^{\dagger} \mathbf{K}_{z}^{-1}\right],
$$

and if the channel is detected as idle, the transmission rate is

$$
r_{2}=B \max _{\substack{\mathbf{K}_{x_{2}} \succeq 0 \\ \operatorname{tr}\left(\mathbf{K}_{x_{2}}\right) \leq 1}} \log _{2} \operatorname{det}\left[\mathbf{I}+\nu N \operatorname{SNR} \mathbf{H} \mathbf{K}_{x_{2}} \mathbf{H}^{\dagger}\right] .
$$

In scenarios 1 and 4 , transmission occurs at the rate of instantaneous channel capacity, i.e., $r_{1}=C_{1}$ in scenario 1 and $r_{2}=C_{4}$ in scenario 4. In scenario 3 , channel is detected as busy despite its being idle. In this case, channel is not fully utilized and the transmission rate is less than the capacity, i.e., 


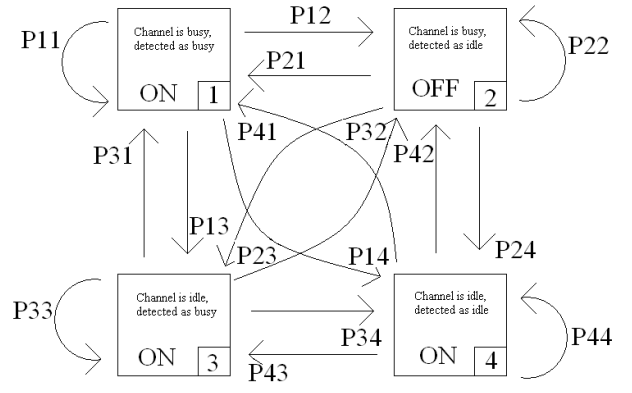

Fig. 1. State transition model for the cognitive radio channel. The numbered label for each state is given on the lower-right corner of the box representing the state.

$r_{1} \leq C_{3}$. In all of the above-mentioned scenarios, communication is performed reliably. On the other hand, in scenario 2, we have the transmission rate exceeding the channel capacity $\left(r_{2}>C_{2}\right)$ because sensing has not detected the active primary users successfully and their interference on the secondary users' signals are not taken into account. In this case, we assume that outage occurs and communication fails. Hence, the transmission rate is effectively zero, and retransmission is required. These four scenarios or equivalently states are depicted in Figure 1. As described above, the channel is $\mathrm{ON}$ in states 1,2, and 4 and OFF in state 2.

Next, we determine the state-transition probabilities. We use $p_{i j}$ to denote the transition probability from state $i$ to state $j$ as seen in Fig. 1. Due to the block fading assumption, state transitions occur every $T$ seconds. The transition probabilities depend only on the detection probabilities and the prior probability of the channel being busy, $\rho$. These probabilities can be expressed as

$$
\begin{aligned}
& p_{i 1}=p_{1}=\rho P_{d}, \quad p_{i 2}=p_{2}=\rho\left(1-P_{d}\right), \\
& p_{i 3}=p_{3}=(1-\rho) P_{f}, \quad \text { and } \quad p_{i 4}=p_{4}=(1-\rho)\left(1-P_{f}\right), \\
& \text { for } i=1,2,3,4 .
\end{aligned}
$$

\section{B. Effective Capacity}

The effective capacity for a given QoS exponent $\theta$ is [4]

$$
-\lim _{t \rightarrow \infty} \frac{1}{\theta t} \log _{e} \mathbb{E}\left\{e^{-\theta S(t)}\right\}=-\frac{\Lambda(-\theta)}{\theta}
$$

where $\Lambda(\theta)=\lim _{t \rightarrow \infty} \frac{1}{t} \log _{e} \mathbb{E}\left\{e^{\theta S(t)}\right\}$ is a function that depends on the logarithm of the moment generating function of $S(t)$, where $S(t)=\sum_{k=1}^{t} r(k)$ is the time-accumulated service process, and $\{r(k), k=1,2, \ldots\}$ is defined as the discrete-time, stationary and ergodic stochastic service process. The QoS exponent $\theta$ provides the decay rate of the buffer violation probability for large buffer limits, i.e., for large $Q_{\max }$,
$P\left(Q \geq Q_{\max }\right) \approx e^{-\theta Q_{\max }}$ where $Q$ is the stationary queue length (see [13] and [14] for more details). Hence, large $\theta$ corresponds to more stringent buffer constraints.

Note that the service rate in $k^{\text {th }}$ transmission block is $r(k)=$ $T r_{1}$ if the cognitive system is in state (or equivalently scenario) 1 or 3. Similarly, the service rate is $r(k)=T r_{2}$ in state 4 . In state 2 , the service rate is effectively zero. Considering the transmission rates in each states and the probabilities of these states, we obtain the following result.

Theorem 1: For the cognitive radio channel with the aforementioned state transition model, the normalized effective capacity in bits/s/Hz/dimension is given by

$$
\begin{aligned}
C_{E}(\mathrm{SNR}, \theta)= & \max _{\substack{\mu, \nu \geq 0 \\
P_{d} \mu+\left(1-P_{d}\right) \nu \leq 1}}-\frac{1}{\theta T B N} \log _{e} \mathbb{E}\left\{\left[p_{1}+p_{3}\right] e^{-\theta T r_{1}}\right. \\
& \left.+p_{4} e^{-\theta T r_{2}}+p_{2}\right\} \text { bits/s/Hz/dimension }
\end{aligned}
$$

where $T$ is the frame duration over which the fading stays constant, $r_{1}$ and $r_{2}$ are the transmission rates, and $\left\{p_{i}\right\}_{i=1}^{4}$ are the state transition probabilities.

Note that, above, we have assumed that $\mathbf{H}$ and $\mathbf{K}_{z}$ are perfectly known at the transmitter. If, on the other hand, only statistical information related to $\mathbf{H}$ are known at the transmitter, then the input covariance matrix can be chosen to maximize the effective capacity. In that case, the normalized effective capacity will be expressed as

$$
\begin{aligned}
& C_{E}(\mathrm{SNR}, \theta)=\max _{\substack{\mu, \nu \geq 0 \\
P_{d} \mu+\left(1-P_{d} \nu \leq 1\right.}} \max _{\mathbf{t r}\left(\mathbf{K}_{x_{1}}\right), \mathbf{t r}\left(\mathbf{K}_{x_{2}}\right) \leq 1}-\frac{1}{\theta T B N} \log _{e} \\
& \mathbb{E}\left\{\left[p_{1}+p_{3}\right] e^{-\theta T B \log _{2} \operatorname{det}\left[\mathbf{I}+\mu N \operatorname{SNR} \mathbf{H} \mathbf{K}_{x_{1}} \mathbf{H}^{\dagger} \mathbf{K}_{z}^{-1}\right]}\right. \\
& \left.+p_{4} e^{-\theta T B \log _{2} \operatorname{det}\left[\mathbf{I}+\nu N \mathrm{SNR} \mathbf{H} \mathbf{K}_{x_{2}} \mathbf{H}^{\dagger}\right]}+p_{2}\right\} \text { bits/s/Hz/dimension. }
\end{aligned}
$$

For given input covariance matrices $\mathbf{K}_{x_{1}}$ and $\mathbf{K}_{x_{2}}$, and for given $\mu$ and $\nu$, we express the effective rate as

$$
\begin{aligned}
& R_{E}\left(P_{1}, P_{2}, \theta\right)=-\frac{1}{\theta T B N} \log _{e} \\
& \mathbb{E}\left\{\left[\rho P_{d}+(1-\rho) P_{f}\right] e^{-\theta T B \log _{2} \operatorname{det}\left[\mathbf{I}+\mu N \operatorname{sNRHK}_{x_{1}} \mathbf{H}^{\dagger} \mathbf{K}_{z}^{-1}\right]}\right. \\
& +(1-\rho)\left(1-P_{f}\right) e^{-\theta T B \log _{2} \operatorname{det}\left[\mathbf{I}+\nu N \operatorname{sNR} \mathbf{H K} \mathbf{K}_{2} \mathbf{H}^{\dagger}\right]} \\
& \left.+\rho\left(1-P_{d}\right)\right\} \text { bits/s/Hz/dimension. }
\end{aligned}
$$

\section{Ergodic Capacity}

As $\theta$ vanishes, the QoS constraints become loose and it can be easily verified that the effective capacity approaches the ergodic 
channel capacity, i.e.,

$$
\begin{aligned}
& \lim _{\theta \rightarrow 0} C_{E}(\mathrm{SNR}, \theta)=\frac{1}{N} \max _{\substack{\mu, \nu \geq 0 \\
P_{d} \mu+\left(1-P_{d}\right) \nu \leq 1}} \\
& \mathbb{E}\left\{\left[p_{1}+p_{3}\right] \max _{\substack{\mathbf{K}_{x_{1}} \succeq 0 \\
\operatorname{tr}\left(\mathbf{K}_{x_{1}}\right) \leq 1}} \log _{2} \operatorname{det}\left[\mathbf{I}+\mu N \operatorname{SNRHK}_{x_{1}} \mathbf{H}^{\dagger} \mathbf{K}_{z}^{-1}\right]\right. \\
& \left.+p_{4} \max _{\substack{\mathbf{K}_{x_{2}} \succeq 0 \\
\operatorname{tr}\left(\mathbf{K}_{x_{2}}\right) \leq 1}} \log _{2} \operatorname{det}\left[\mathbf{I}+\nu N \operatorname{SNR} \mathbf{H K} \mathbf{K}_{x_{2}} \mathbf{H}^{\dagger}\right]\right\}
\end{aligned}
$$

\section{EFFECTIVE CAPACITy IN THE LOW-Power Regime}

\section{A. First and Second Derivatives of the Effective Capacity}

In this section, we study the effective capacity in the lowSNR regime and investigate the impact of the QoS constraints. The analysis is conducted for given values of $\mu$ and $\nu$. First, we consider the following second-order expansion of the effective capacity:

$$
C_{E}(\mathrm{SNR}, \theta)=\dot{C}_{E}(0, \theta) \mathrm{SNR}+\ddot{C}_{E}(0, \theta) \frac{\mathrm{SNR}^{2}}{2}+o\left(\mathrm{SNR}^{2}\right)
$$

where $\dot{C}_{E}(0, \theta)$ and $\ddot{C}_{E}(0, \theta)$ denote the first and second derivatives of the effective capacity with respect to SNR at $\mathrm{SNR}=0$. We can first show that

$$
\dot{R}_{E}(0, \theta) \leq \frac{a \mu \mathbb{E}\left[\lambda_{\max }\left(\mathbf{H}^{\dagger} \mathbf{K}_{z}^{-1} \mathbf{H}\right)\right]+b \nu \mathbb{E}\left[\lambda_{\max }\left(\mathbf{H}^{\dagger} \mathbf{H}\right)\right]}{\log _{e} 2}
$$

where $\lambda_{\max }\left(\mathbf{H}^{\dagger} \mathbf{K}_{z}^{-1} \mathbf{H}\right)$ and $\lambda_{\max }\left(\mathbf{H}^{\dagger} \mathbf{H}\right)$ denote the maximum eigenvalues of the matrices $\mathbf{H}^{\dagger} \mathbf{K}_{z}^{-1} \mathbf{H}$ and $\mathbf{H}^{\dagger} \mathbf{H}$. The upper bound in (21) can be achieved by choosing the normalized input covariance matrices as

$$
\mathbf{K}_{x_{1}}=\mathbf{u}_{1} \mathbf{u}_{1}^{\dagger} \quad \text { and } \quad \mathbf{K}_{x_{2}}=\mathbf{u}_{2} \mathbf{u}_{2}^{\dagger}
$$

where $\mathbf{u}_{1}$ and $\mathbf{u}_{2}$ are the unit-norm eigenvectors that correspond to the maximum eigenvalues $\lambda_{\max }\left(\mathbf{H}^{\dagger} \mathbf{K}_{z}^{-1} \mathbf{H}\right)$ and $\lambda_{\max }\left(\mathbf{H}^{\dagger} \mathbf{H}\right)$. This lets us conclude that

$$
\dot{C}_{E}(0, \theta)=\frac{a \mu \mathbb{E}\left[\lambda_{\max }\left(\mathbf{H}^{\dagger} \mathbf{K}_{z}^{-1} \mathbf{H}\right)\right]+b \nu \mathbb{E}\left[\lambda_{\max }\left(\mathbf{H}^{\dagger} \mathbf{H}\right)\right]}{\log _{e} 2} .
$$

We note that more generally, if the maximum eigenvalues $\lambda_{\max }\left(\mathbf{H}^{\dagger} \mathbf{K}_{z}^{-1} \mathbf{H}\right)$ and $\lambda_{\max }\left(\mathbf{H}^{\dagger} \mathbf{H}\right)$ have multiplicities of $l_{1} \geq$ 1 and $l_{2} \geq 1$, respectively, the input-covariance matrices optimal in the sense of attaining $\dot{C}_{E}(0, \theta)$ can be written in the following forms:

$$
\mathbf{K}_{x_{1}}=\sum_{i=1}^{l_{1}} \kappa_{1 i} \mathbf{u}_{1, i} \mathbf{u}_{1, i}^{\dagger} \quad \text { and } \quad \mathbf{K}_{x_{2}}=\sum_{i=1}^{l_{2}} \kappa_{2 i} \mathbf{u}_{2, i} \mathbf{u}_{2, i}^{\dagger}
$$

where $\kappa_{1 i}, \kappa_{2 i} \in[0,1]$ and $\sum_{i=1}^{l_{1}} \kappa_{1 i}=1$ and $\sum_{i=1}^{l_{2}} \kappa_{2 i}=1$, and $\left\{\mathbf{u}_{1, i}\right\}$ and $\left\{\mathbf{u}_{2, i}\right\}$ are the orthonormal eigenvectors that span the maximal-eigenvalue eigenspaces of $\mathbf{H}^{\dagger} \mathbf{K}_{z}^{-1} \mathbf{H}$ and $\mathbf{H}^{\dagger} \mathbf{H}$, respectively.

With the above optimal covariance matrices, we can easily verify that

$$
\begin{aligned}
& \mathbb{E}\left\{\operatorname{tr}\left(\Phi_{1}\right)\right\}=\mathbb{E}\left\{\operatorname{tr}\left(\mathbf{H K}_{x_{1}} \mathbf{H}^{\dagger} \mathbf{K}_{z}^{-1}\right)\right\}=\mathbb{E}\left\{\lambda_{\max }\left(\mathbf{H}^{\dagger} \mathbf{K}_{z}^{-1} \mathbf{H}\right)\right\} \\
& \mathbb{E}\left\{\operatorname{tr}\left(\Phi_{2}\right)\right\}=\mathbb{E}\left\{\operatorname{tr}\left(\mathbf{H K}_{x_{2}} \mathbf{H}^{\dagger}\right)\right\}=\mathbb{E}\left\{\lambda_{\max }\left(\mathbf{H}^{\dagger} \mathbf{H}\right)\right\}
\end{aligned}
$$

and

$$
\begin{aligned}
& \mathbb{E}\left\{\operatorname{tr}\left(\mathbf{K}_{z}^{-1} \mathbf{H K}_{x_{1}} \mathbf{H}^{\dagger} \mathbf{H} \mathbf{K}_{x_{1}} \mathbf{H}^{\dagger} \mathbf{K}_{z}^{-1}\right)\right\} \geq \frac{1}{l_{1}} \mathbb{E}\left\{\lambda_{\max }^{2}\left(\mathbf{H}^{\dagger} \mathbf{K}_{z}^{-1} \mathbf{H}\right)\right\} \\
& \mathbb{E}\left\{\operatorname{tr}\left(\mathbf{H K}_{x_{1}} \mathbf{H}^{\dagger} \mathbf{H} \mathbf{K}_{x_{1}} \mathbf{H}^{\dagger}\right)\right\} \geq \frac{1}{l_{2}} \mathbb{E}\left\{\lambda_{\max }^{2}\left(\mathbf{H}^{\dagger} \mathbf{H}\right)\right\}
\end{aligned}
$$

Using these results, we obtain the following upper bound for the second derivative of effective rate, which can be achieved and hence is equal to $\ddot{C}_{E}(0, \theta)$ :

$$
\begin{aligned}
& \ddot{R}_{E}(0, \theta) \leq \frac{\theta T B N}{\log _{e}^{2} 2} \mathbb{E}^{2}\left[a \mu \lambda_{\max }\left(\mathbf{H}^{\dagger} \mathbf{K}_{z}^{-1} \mathbf{H}\right)+b \nu \lambda_{\max }\left(\mathbf{H}^{\dagger} \mathbf{H}\right)\right] \\
& -\frac{\theta T B N}{\log _{e}^{2} 2} \mathbb{E}\left[a \mu^{2} \lambda_{\max }^{2}\left(\mathbf{H}^{\dagger} \mathbf{K}_{z}^{-1} \mathbf{H}\right)+b \nu^{2} \lambda_{\max }^{2}\left(\mathbf{H}^{\dagger} \mathbf{H}\right)\right] \\
& -\frac{N}{\log _{e} 2} \mathbb{E}\left[\frac{a \mu^{2} \lambda_{\max }^{2}\left(\mathbf{H}^{\dagger} \mathbf{K}_{z}^{-1} \mathbf{H}\right)}{l_{1}}+\frac{b \nu^{2} \lambda_{\max }^{2}\left(\mathbf{H}^{\dagger} \mathbf{H}\right)}{l_{2}}\right] \\
& =\ddot{C}_{E}(0, \theta)
\end{aligned}
$$

\section{B. Energy Efficiency in the Low-Power Regime}

Now, we can analyze the energy efficiency in the low-power regime using the expressions for the first and second derivatives. The minimum bit energy under QoS constraints is given by [7], [16]

$$
\frac{E_{b}}{N_{0} \text { min }}=\lim _{\mathrm{SNR} \rightarrow 0} \frac{\mathrm{SNR}}{C_{E}(\mathrm{SNR})}=\frac{1}{\dot{C}_{E}(0)} .
$$

At $\frac{E_{b}}{N_{0 \text { min }}}$, the slope $\mathcal{S}_{0}$ of the spectral efficiency versus $E_{b} / N_{0}$ (in $\mathrm{dB}$ ) curve is defined as [16]

$$
\mathcal{S}_{0}=\lim _{\frac{E_{b}}{N_{0}} \downarrow \frac{E_{b}}{N_{0}}{ }_{\text {min }}} \frac{C_{E}\left(\frac{E_{b}}{N_{0}}\right)}{10 \log _{10} \frac{E_{b}}{N_{0}}-10 \log _{10} \frac{E_{b}}{N_{0}} \min } 10 \log _{10} 2 .
$$

Considering the expression for normalized effective capacity, the wideband slope can be found from [16]

$\mathcal{S}_{0}=\frac{2\left[\dot{C}_{E}(0)\right]^{2}}{-\ddot{C}_{E}(0)} \log _{e} 2 \quad$ bits $/ \mathrm{s} / \mathrm{Hz} /(3 \mathrm{~dB}) /$ receive antenna.

Applying the results (23) and (27) to the above formulation, we obtain

$$
\begin{gathered}
{\frac{E}{N_{0}}}_{\min }=\frac{\log _{e} 2}{a \mu \mathbb{E}\left[\lambda_{\max }\left(\mathbf{H}^{\dagger} \mathbf{K}_{z}^{-1} \mathbf{H}\right)\right]+b \nu \mathbb{E}\left[\lambda_{\max }\left(\mathbf{H}^{\dagger} \mathbf{H}\right)\right]} \\
\mathcal{S}_{0}=\frac{2 \mathbb{E}^{2}[a \mu \alpha+b \nu \beta]}{A}
\end{gathered}
$$

where $A=\theta T B N\left\{\mathbb{E}\left[a \mu^{2} \alpha^{2}+b \nu^{2} \beta^{2}\right]-\mathbb{E}^{2}[a \mu \alpha+b \nu \beta]\right\}+$ $N \mathbb{E}\left[\frac{a \mu^{2} \alpha^{2}}{l_{1}}+\frac{b \nu^{2} \beta^{2}}{l_{2}}\right] \log _{e} 2, \alpha=\lambda_{\max }\left(\mathbf{H}^{\dagger} \mathbf{K}_{z}^{-1} \mathbf{H}\right)$ and $\beta=$ $\lambda_{\max }\left(\mathbf{H}^{\dagger} \mathbf{H}\right)$.

When we have equal power allocation, i.e., $\mathbf{K}_{x}=\frac{1}{M} \mathbf{I}$, and with the assumption that $\mathbf{s}$ with dimension $N \times 1$ is a zero-mean Gaussian random vector with a covariance matrix $\mathbb{E}\left\{\mathbf{s s}^{\dagger}\right\}=\sigma_{s}^{2} \mathbf{I}$ 

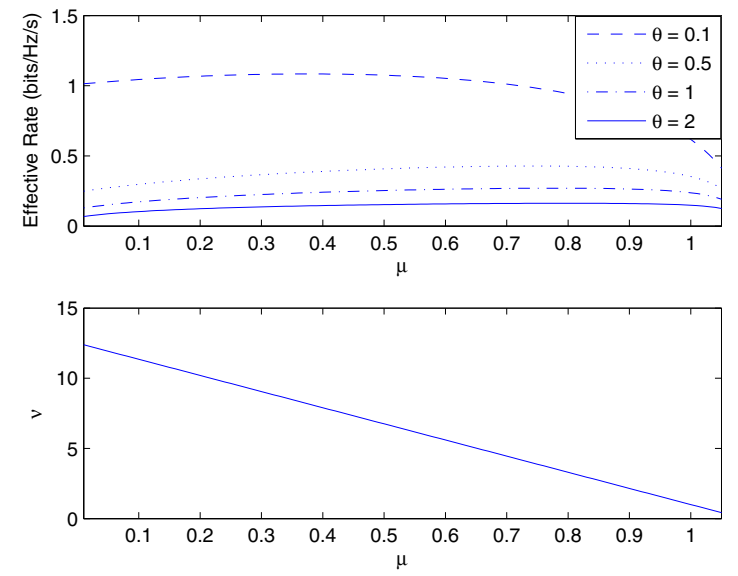

Fig. 2. Effective Rate and $\nu$ v.s. $\mu$ for different Decay Rate, $\theta$, values.

where $\mathbf{I}$ is the identity matrix, it can be immediately seen from (31) and (32) that

$$
\begin{aligned}
& {\frac{E_{b}}{N_{0} \text { min }}}=\frac{\log _{e} 2}{\left(\frac{a \mu}{\sigma_{s}^{2}}+b \nu\right) \mathbb{E}\left[\operatorname{tr}\left(\mathbf{H}^{\dagger} \mathbf{H}\right)\right]} \\
& \mathcal{S}_{0}=\frac{2\left(\frac{a \mu}{\sigma_{s}^{2}}+b \nu\right)^{2} \mathbb{E}^{2}\left[\operatorname{tr}\left(\mathbf{H}^{\dagger} \mathbf{H}\right)\right]}{A}
\end{aligned}
$$

where $A=\theta T B N\left\{\left[\frac{a \mu^{2}}{\sigma_{s}^{4}}+b \nu^{2}\right] \mathbb{E}\left[\operatorname{tr}^{2}\left(\mathbf{H}^{\dagger} \mathbf{H}\right)\right]-\left[\frac{a \mu}{\sigma_{s}^{2}}+\right.\right.$ $\left.b \nu]^{2} \mathbb{E}^{2}\left[\operatorname{tr}\left(\mathbf{H}^{\dagger} \mathbf{H}\right)\right]\right\}+N\left[\frac{a \mu^{2}}{\sigma_{s}^{4}}+b \nu^{2}\right] \mathbb{E}\left[\operatorname{tr}\left(\left(\mathbf{H}^{\dagger} \mathbf{H}\right)^{2}\right)\right] \log _{e} 2$. Now, assuming that $\mathbf{H}$ has independent zero-mean unit-variance complex Gaussian random entries, we have [2]

$$
\begin{aligned}
& \mathbb{E}\left[\operatorname{tr}\left(\mathbf{H}^{\dagger} \mathbf{H}\right)\right]=N M, \quad \mathbb{E}\left[\operatorname{tr}^{2}\left(\mathbf{H}^{\dagger} \mathbf{H}\right)\right]=N M(N M+1), \\
& \text { and } \mathbb{E}\left[\operatorname{tr}\left(\left(\mathbf{H}^{\dagger} \mathbf{H}\right)^{2}\right)\right]=N M(N+M) .
\end{aligned}
$$

Using these facts, we can write the following minimum bit energy and wideband slope expressions for the uniform power allocation case

$$
{\frac{E_{b}}{N_{0} \text { min }}}=\frac{\log _{e} 2}{\left(\frac{a \mu}{\sigma_{s}^{2}}+b \nu\right) N M} \quad \text { and } \quad \mathcal{S}_{0}=\frac{2\left(\frac{a \mu}{\sigma_{s}^{2}}+b \nu\right)^{2} M^{2}}{A}
$$

$A=\theta T B\left\{\left[\frac{a \mu^{2}}{\sigma_{s}^{4}}+b \nu^{2}\right] M(N M+1)-\left[\frac{a \mu}{\sigma_{s}^{2}}+b \nu\right]^{2} M^{2}\right\}+$ $\left[\frac{a \mu^{2}}{\sigma_{s}^{4}}+b \nu^{2}\right] M(N+M) \log _{e} 2$.

It is interesting to note that in all cases, $\frac{E_{b}}{N_{0}}$ min does not depend on the QoS exponent $\theta$ while $\mathcal{S}_{0}$ decreases with increasing $\theta$.

\section{Numerical Results}

In this section, we numerically show the results obtained in the previous sections. In our simulations, we consider the independent and identically distributed (i.i.d.) Rayleigh fading

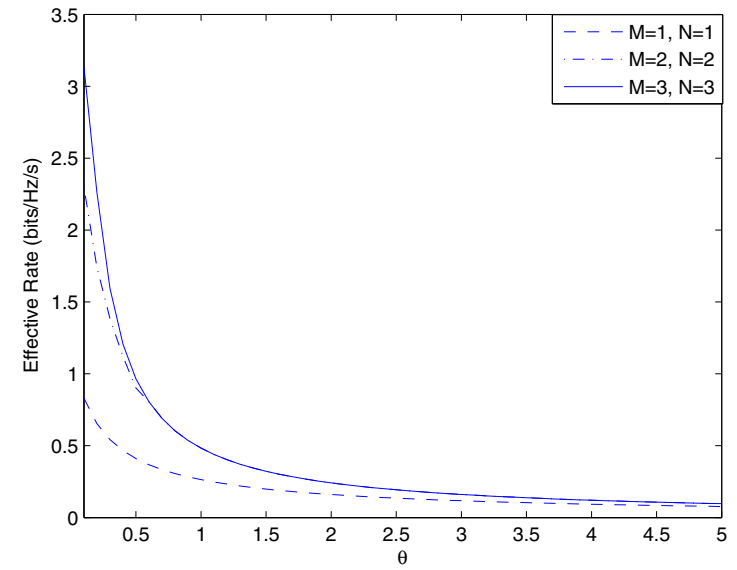

Fig. 3. Effective Rate v.s. Decay Rate, $\theta$ for different Number of Antennas, M.

channel for which the components of the channel matrix $\mathbf{H}$ are i.i.d. zero-mean, unit variance, circularly symmetric Gaussian random variables. Moreover, we assume that input covariance matrix is $\mathbf{K}_{x}=\frac{1}{M} \mathbf{I}$ and that components of received signal coming from primary users are i.i.d. and have variance $\sigma_{s}^{2}$ so that $\mathbf{K}_{z}=\frac{\sigma_{s}^{2}+\sigma_{n}^{2}}{\sigma_{s}^{2}} \mathbf{I}$. We consider the effective rate which is given by

$$
\begin{aligned}
& R_{E}(\mathrm{SNR}, \theta)=-\frac{1}{\theta T B} \log _{e} \\
& \mathbb{E}\left\{a e^{-\theta T B \log _{2} \operatorname{det}\left[\mathbf{I}+\frac{\mu N \sigma_{n}^{2}}{M\left(\sigma_{s}^{2}+\sigma_{n}^{2}\right)} \mathrm{SNRHH}^{\dagger}\right]}\right. \\
& \left.+b e^{-\theta T B \log _{2} \operatorname{det}\left[\mathbf{I}+\frac{\nu N}{M} \mathrm{SNRHH} H^{\dagger}\right]}+\rho\left(1-P_{d}\right)\right\} \mathrm{bits} / \mathrm{Hz} / \mathrm{s} .
\end{aligned}
$$

With these assumptions, we calculate the effective rate by using the expression for the moment generating function of instantaneous mutual information given by Wang and Giannakis in [17, Theorem 1]. After adopting this expression in our effective rate formulation (37), we obtain

$$
\begin{aligned}
& R_{E}(\mathrm{SNR}, \theta)=-\frac{1}{\theta T B} \log _{e} \\
& \left\{\left[\rho P_{d}+(1-\rho) P_{f}\right] \frac{\operatorname{det}\left[\mathbf{G}\left(\theta, \frac{\mu \sigma_{n}^{2} \mathrm{SNR}}{\sigma_{s}^{2}+\sigma_{n}^{2}}\right)\right]}{\prod_{i=1}^{k} \Gamma(d+i)}\right. \\
& \left.+(1-\rho)\left(1-P_{f}\right) \frac{\operatorname{det}[\mathbf{G}(\theta, \nu \mathrm{SNR})]}{\prod_{i=1}^{k} \Gamma(d+i)}+\rho\left(1-P_{d}\right)\right\} \mathrm{bits} / \mathrm{Hz} / \mathrm{s}
\end{aligned}
$$

where $k=\min (M, N), d=\max (M, N)-\min (M, N)$, and $\Gamma($.$) is the Gamma function. Here, \mathbf{G}(\theta$, SNR $)$ is a $k \times k$ Hankel matrix whose $(m, n)^{t h}$ component is

$$
\begin{gathered}
g_{m, n}=\int_{0}^{\infty}\left(1+\frac{N}{M} \mathrm{SNR} z\right)^{-\theta T B \log _{2} e} z^{m+n+d-2} e^{-z} d z \\
m, n=1,2, \ldots, k .
\end{gathered}
$$

In our simulations, we assume $T=0.1 \mathrm{~s}, B=100 \mathrm{~Hz}$, 


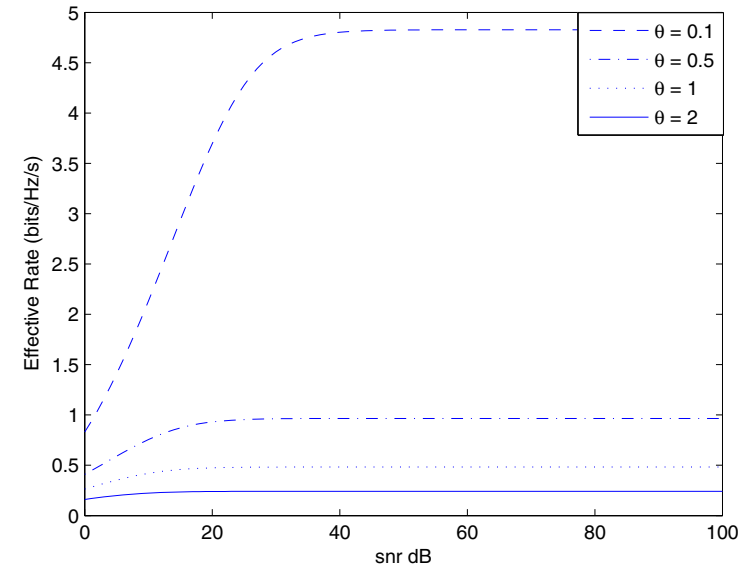

Fig. 4. Effective Rate v.s. SNR for different values of Decay Rate, $\theta \mathrm{M}=1$.

$\sigma_{n}^{2}=\sigma_{s}^{2}=1, \rho=0.1, P_{d}=0.92$ and $P_{f}=0.21$. In Fig. 2, we plot the effective rate as a function of $\mu$ for different values of the QoS exponent $\theta$. As expected, with higher values of $\theta$, QoS constraints become more stringent and the effective rate decreases. We also notice that the maximum effective rate is achieved at a higher value of $\mu$ when $\theta$ has a larger value, meaning that under more stringent buffer constraints, more power is allocated for the case in which the primary users are active. In this figure, the case of single transmit and single receive antenna is considered and it is assumed that $\mathrm{SNR}=0 d B$. In Figure 3, we plot the effective rate as a function of $\theta$ for different values of transmit and receive antennas when, again, $\mathrm{SNR}=0 d B$. We note that the effective rate is decreasing in all cases as $\theta$ values are increased. We also observe that gains achieved by having multiple antennas diminish for high values of $\theta$. In Figs. 4 and 5, we display the effective rate as a function of SNR for different values of $\theta$. In Fig. 4, transmitter and receiver are each equipped with a single antenna whereas in Fig. 5 we assume 3-transmit and 3-receive antennas. It can be easily observed that at high SNR values, the number of antennas does not contribute much to the effective rates. On the other hand, using more antennas at lower SNR values is more beneficial for the secondary users under strict buffer constraints.

\section{CONCLUSION}

In this paper, we have investigated the performance levels of cognitive MIMO wireless communication systems under queuing constraints. We have determined the effective capacity of the MIMO cognitive channel. We have studied the performance in the low-power regime. We have obtained the expressions for the first and second derivatives of effective capacity. We have observed that the first derivative does not depend on $\theta$ but it is affected by the power allocation strategies for the case when the channel is busy and the case when the channel is idle. On the other hand, the second derivative is a function of the QoS exponent $\theta$. We have also determined the minimum bit energy requirements under the QoS constraints.

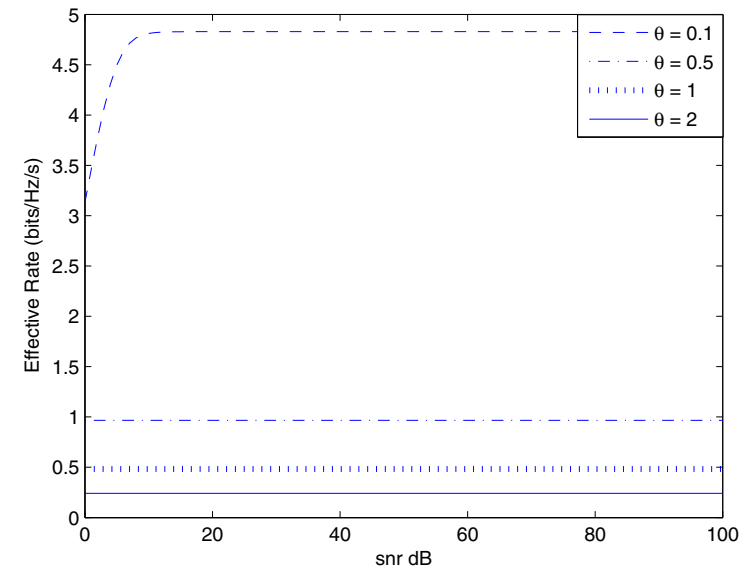

Fig. 5. Effective Rate v.s. SNR for different values of Decay Rate, $\theta \mathrm{M}=3$.

\section{REFERENCES}

[1] A. Goldsmith, S. A. Jafar, N. Jindal, and S. Vishwanath, "Capacity limits of MIMO channels," IEEE Journ. on Select. Areas in Commun., vol. 21, No. 5, PP. 684-702, Jun. 2003.

[2] A. Lozano, A. M. Tulino, and S. Verdu "Multiple-antenna capacity in the low-power regime," IEEE Trans. Inform. Theory, vol. 49, no. 10, pp.25272544, Oct. 2003.

[3] A. Lozano and A. M. Tulino, "Capacity of multiple-transmit multiplereceive antenna architectures," IEEE Trans. on Inform. Theory, Vol. 48, No. 12, Dec. 2002.

[4] D. Wu and R. Negi, "Effective capacity: A wireless link model for support of quality of service," IEEE Trans. Wireless Commun., vol. 2, no. 4, pp. 630-643. July 2003.

[5] E. A. Jorswieck, R. Mochaourab, and M. Mittelbach, "Effective capacity maximization in multi-antenna channels with covariance feedback," IEEE Int. Conf. on Commun., Dresden Germany, 2009.

[6] L. Liu, and J.-F. Chamberland, "On the effective capacities of multipleantenna Gaussian channels," IEEE International Symposium on Information Theory, Toronto, 2008.

[7] M. C. Gursoy, "MIMO wireless communications under statistical queuing constraints," submitted to the IEEE Transactions on Information Theory, Sept. 2009

[8] A. Goldsmith, S. A. Jafar, I ,Maric, and S. Srinivasa, "Breaking spectrum gridlock with cognitive radios: An information theoretic perspective," Proc. of the IEEE, Vol. 97, No. 5, May 2009.

[9] Q. Zhao and B. Sadler, "A survey of dynamic spectrum access," IEEE Sign. Process. Mag., Vol. 24, No. 3, PP. 79-89, May 2007.

[10] R. Zhang and Y. C. Liang, "Exploiting multi-antennas for opportunistic spectrum sharing in cognitive radio networks," IEEE Journal of Selected Topics in Signal Processing, vol. 2, no. 1, pp. 88-102, February 2008.

[11] Y. Chen, Q. Zhao, and A. Swami, "Joint design and separation principle for opportunistic spectrum access in the presence of sensing errors," IEEE Trans. Inform. Theory, Vol. 54, PP. 2053-2071, May 2008.

[12] Q. Zhao, "Spectrum opportunity and interference constraint in opportunistic spectrum access," in Proc. IEEE Int. Conf. Acoustic, Speech, and Sig. Proc. (ICASSP), Honolulu, HI, PP. III-605-III-608, Apr. 15-20, 2007.

[13] S. Akin and M. C. Gursoy, "Effective capacity analysis of cognitive radio channels for quality of service provisioning," IEEE Trans. on Wire. Comm., vol. 9, no. 11, pp.3354-3364, Nov. 2010.

[14] S. Akin and M. C. Gursoy, "Performance analysis of cognitive radio systems under QoS constraints and channel uncertainty," revised and submitted to the IEEE Trans. on Wire. Comm., Oct. 2010.

[15] R. A. Horn and C. R. Johnson, Matrix Analysis, Cambridge University Press, 1985.

[16] S. Verdú "Spectral efficiency in the wideband regime," IEEE Trans. Inform. Theory, vol.48, no.6, pp.1319-1343, Jun. 2002.

[17] Z. Wang and G. B. Giannakis, "Outage mutual information of space-time MIMO channels," IEEE Trans. Inform. Theory, Vol. 50, No. 4, PP. $657-$ 662, Apr. 2004. 